\title{
Unresectable Digestive System Neuroendocrine Carcinoma
}

National Cancer Institute

\section{Source}

National Cancer Institute. Unresectable Digestive System Neuroendocrine Carcinoma. NCI Thesaurus. Code C155935.

A neuroendocrine carcinoma that arises from any part of the digestive system and is not amenable to surgical resection. 